\title{
Fatores de risco para sibilância recorrente em menores de 13 anos no Sul do Brasil
}

\author{
Silvio Omar Macedo Prietsch, ${ }^{1}$ Gilberto Bueno Fischer, ${ }^{2}$ Juraci Almeida César, ${ }^{1}$ \\ Patrícia Velasques Cervo, ${ }^{3}$ Leonardo Luiz Sangaletti, ${ }^{3}$ Cácio Ricardo \\ Wietzycoski, ${ }^{3}$ Diego Zacca ${ }^{3}$ e Fernanda Martins dos Santos ${ }^{3}$
}

Como citar Prietsch SO, Fischer GB, César JA, Cervo PV, Sangaletti LL, Wietzycoski CR, et al. Fatores de risco para sibilância recorrente em menores de 13 anos no Sul do Brasil. Rev Panam Salud Publica. 2006:20(5): 331-7.

RESUMO Objetivo. Estudar a prevalência de sibilância recorrente e os principais fatores associados em crianças menores de 13 anos da área urbana da Cidade de Rio Grande, Estado do Rio Grande do Sul.

Método. Através de visitas domiciliares e aplicação de questionários padronizados por entrevistadores treinados, investigou-se a presença de sibilância recorrente em uma coorte iniciada em 1997 como parte de um estudo transversal de base populacional que enfocou a morbidade por doenças respiratórias em crianças entre 0 a 5 anos. Foram obtidas informações sobre as condições socioeconômicas e de moradia da família, assistência à gestação e ao parto e padrão de morbidade atual e pregressa das crianças. A análise estatística consistiu no cálculo da razão de chances (odds ratio, OR) com ajuste através de regressão logística não-condicional para potenciais fatores de confusão, conforme modelo hierárquico previamente definido.

Resultados. Das 775 crianças estudadas em 1997, 685 foram localizadas em 2004 (perda de $11,6 \%$ ). Nesse grupo, a prevalência de sibilância recorrente atual foi de $27,9 \%$. Os fatores de risco após a análise ajustada foram: rinite atual (OR $=45,7 ;$ IC95\%:24,2 a 86,5), uso de fogão a lenha para cozinhar $(O R=2,7$; IC95\%: 1,4 a 4,9), antecedente pessoal de infecção respiratória aguda $(O R=2,1 ;$ IC95\%: 1,3 a 3,5), aleitamento artificial $(O R=2,1 ;$ IC95\%: 1,1 a 3,8), antecedente de asma em irmãos (OR =1,9; IC95\%: 1,2 a 3,2), antecedente de asma na mãe $(O R=1,8$; IC95\%: 1,1 a 2,9) e menos de seis consultas de pré-natal $(O R=1,6$; IC95\%: 1,1 a 2,4). Escolaridade do pai $<9$ anos completos representou fator de proteção para sibilância recorrente $(O R=0,6 ;$ IC95\%:0,4 a 0,9$)$.

Conclusão. Os resultados sugerem que o manejo de sibilância recorrente e asma deve contemplar a investigação e o tratamento conjunto de rinite. As ações para minimizar os efeitos da sibilância recorrente devem incluir programas educativos e terapêuticos enfocados na asma.

Palavras-chave Asma, criança, epidemiologia, fatores de risco, sons respiratórios, Brasil.

1 Fundação Universidade Federal do Rio Grande, Departamento Materno-Infantil, Rio Grande (RS), Brasil. Enviar correspondência para Silvio Omar Macedo Prietsch no seguinte endereço: Rua Dr. Nascimento 396, Centro, CEP 96200-300, Rio Grande, RS, Brasil. Fone: +55-53-3232-5431. E-mail: silvio@vetorial.net.
A asma é a principal responsável pelo comprometimento prolongado da

2 Fundação Faculdade Federal de Ciências Médicas de Porto Alegre, Porto Alegre (RS), Brasil.

3 Fundação Universidade Federal do Rio Grande, Faculdade de Medicina, Rio Grande, RS, Brasil. saúde em crianças e adolescentes nos países desenvolvidos e, provavelmente, tem alta prevalência nos países em desenvolvimento (1). Nas últimas décadas, muitos estudos têm mostrado um aumento na prevalência da 
morbidade e maiores taxas de mortalidade por asma em diversos contextos (2). A ocorrência pode variar de menos de $1 \%$ em alguns países em desenvolvimento para mais de $30 \%$ em alguns países desenvolvidos (3).

Um estudo realizado na América Latina (4) mostrou uma prevalência de asma variando de 4,1 a 26,9\% em crianças de 6 a 7 anos. Estudos com escolares em algumas cidades brasileiras (Porto Alegre, São Paulo, Curitiba, Salvador, Recife, Itabira e Uberlândia), realizados como parte do Estudo Internacional de Asma e Alergia na Infância (ISAAC), encontraram taxas de prevalência cumulativa de asma diagnosticada de 7,3\% em meninos e $4,9 \%$ em meninas aos 6 e 7 anos, e de 9,8 e $10,2 \%$, respectivamente, no grupo de 13 e 14 anos $(5,6)$. Um estudo transversal realizado com escolares no Rio de Janeiro encontrou prevalência de $21,7 \%$ de asma de acordo com os critérios do questionário respiratório ATSDLD-78 C modificado (7). Outro estudo, realizado em Pelotas, Estado do Rio Grande do Sul, mostrou prevalência de asma atual diagnosticada por médico de $12,8 \%$ e cumulativa de $31 \%$ (8). Esse mesmo estudo mostrou ainda que $16,8 \%$ das crianças estudadas tiveram crises de chiado nos últimos 12 meses.

O diagnóstico epidemiológico da asma é caracterizado pela ocorrência de três ou mais episódios de sibilância (sibilância recorrente) $(1,2)$. Na Cidade de Rio Grande, próxima de Pelotas, as doenças sibilantes são altamente prevalentes. Uma amostragem representativa da população mostrou que mais de $60 \%$ das crianças menores de 5 anos tiveram pelo menos um episódio de sibilância, e dessas, mais da metade tiveram três ou mais episódios. Um quarto das crianças consultou com médico nos 3 meses anteriores à coleta de dados em decorrência de sibilos e $13 \%$ referiram ter procurado o serviço de saúde por asma (9).

A sibilância recorrente é multicausal e pode estar associada a fatores demográficos, socioeconômicos, genéticos, gestacionais, nutricionais, ambientais e outros. Essa condição tem sido amplamente demonstrada em es- tudos realizados com crianças e adolescentes (8-10). A sibilância recorrente tem um profundo impacto na sociedade e, especialmente, nas crianças e em suas famílias. É responsável por um grande número de consultas médicas de emergência e hospitalizações. Além disso, os custos financeiros diretos e indiretos decorrentes dessa situação são extraordinários (8).

$\mathrm{O}$ presente estudo teve como objetivo medir a ocorrência e identificar os principais fatores associados à sibilância recorrente em uma coorte de crianças menores de 13 anos na Cidade de Rio Grande, Brasil. Essa coorte havia sido avaliada inicialmente em 1997 como parte de um estudo transversal de base populacional que enfocou a morbidade por doenças respiratórias em crianças com idade entre 0 a 5 anos.

\section{MATERIAIS E MÉTODOS}

A Cidade de Rio Grande está localizada na planície costeira do extremo sul do Brasil. Possui área de $3338 \mathrm{~km}^{2}$ e cerca de 200000 habitantes. O clima é subtropical marítimo, com as quatro estações do ano bem definidas e temperaturas que variam de $0^{\circ} \mathrm{C}$ no inverno a $38^{\circ} \mathrm{C}$ no verão. Sua economia está baseada nas atividades portuária, pesqueira e industrial. $O$ parque industrial é constituído por unidades químicas de grande porte e por uma refinaria de petróleo.

Para o estudo realizado em 1997, o cálculo da amostra foi feito para alcançar uma precisão de 5 pontos percentuais, com um nível de confiança de $95 \%$ e um poder de $80 \%$. Com base nesses cálculos, seria necessário estudar 771 crianças, já incluídos aqui $10 \%$ para eventuais perdas e $15 \%$ para controle de fatores de confusão (9). Utilizou-se amostragem sistemática por conglomerados, feita com base nos setores censitários do Instituto Brasileiro de Geografia e Estatística (IBGE). Um setor censitário corresponde a um conjunto de 200 a 300 domicílios previamente demarcados pelo IBGE. Decidiu-se a priori que o estudo cobriria um terço dos setores censitários lo- calizados na zona urbana, o que correspondia a 66 setores (198/3). Os dados do IBGE permitiam estimar a existência de uma criança menor de 5 anos a cada quatro domicílios. Assim, para atingir o tamanho amostral desejado, foi necessário visitar 47 domicílios por setor (número de domicílios por setor $=771 / 0,25 \times 66)$. Setecentas e setenta e cinco crianças foram identificadas em 3185 domicílios visitados.

Em janeiro de 2004, as mesmas crianças foram novamente procuradas para uma visita. Seis bolsistas cedidos pela Prefeitura Municipal de Rio Grande foram treinados para pesar e medir a estatura das crianças e aplicar questionários padronizados às mães ou pessoas responsáveis. Foram utilizados dois tipos de questionário: um buscava informações sobre as características das mães e famílias, e o outro, sobre as características da criança. Foram levantadas informações sobre aspectos demográficos e reprodutivos maternos, condições de saneamento e moradia, nível socioeconômico, utilização dos serviços de saúde, antecedentes pessoais para doenças respiratórias e sibilância, amamentação, estatura, peso atual e ao nascer e antecedentes familiares de doença respiratória. As questões referentes ao histórico de doença pulmonar e à avaliação do aparelho respiratório foram desenvolvidas tendo como base o questionário ATS-DLD-78-C (11).

A sibilância recorrente foi definida pela ocorrência de três ou mais episódios de sibilos, identificados pela mãe ou pessoa responsável pela guarda da criança como "chiado no peito", com uso de broncodilatador ou antiinflamatório por via oral, parenteral ou tópica (inalatória), acompanhados ou não de taquipnéia, dificuldade ventilatória ou tosse seca. Foram estudadas variáveis demográficas (sexo, idade e cor da pele da criança), maternas (cor da pele, idade atual e por ocasião do parto), socioeconômicas (renda familiar e escolaridade dos pais), condições de moradia e saneamento (aglomeração, tipo de construção da casa e do piso, abastecimento de água e instalações sanitárias, presença de animais domésticos e fumo no domicílio), variáveis nutricionais 
(peso de nascimento, estado nutricional e aleitamento materno), antecedentes familiares de asma e rinite e antecedentes pessoais de doenças respiratórias, asma e rinite.

Utilizou-se o programa Epi-Info 6.02 para entrada dos dados, comparação de digitações e análise de consistência. O Statistical Package for the Social Sciences (SPSS) para Windows, versão 8.0, foi utilizado para as análises bi e multivariada.

O plano de análise incluiu quatro etapas:

- obtenção de listagem de freqüências de todas as variáveis (desfecho, fatores de risco e fatores de confusão) e exame das suas distribuições. Quando possível, foram verificadas as medidas de tendência central (média, mediana e moda) e de dispersão (amplitude e desvio padrão);

- recodificação, quando necessário, com definição de pontos de corte para fatores de risco e confusão;

- cruzamento de variáveis independentes com o desfecho (sibilância recorrente). A magnitude das associações foi estimada através da razão de chances (odds ratio, OR) e seu intervalo de confiança de 95\% (IC95\%). Para calcular a significância estatística das associações foi utilizado o teste do qui-quadrado $\left(\chi^{2}\right)$ de Pearson, com a correção de Yates para variáveis com apenas duas categorias. Para variáveis com mais de duas categorias e ordenação lógica, utilizou-se o teste para tendência linear em proporções;

- análise multivariada com controle simultâneo para diversos fatores de risco ou confusão, através do método de regressão logística nãocondicional para obtenção de OR e aplicação do teste de razão de verossimilhança (TRV) para significância estatística (12). Essa análise levou em consideração um modelo hierarquizado em que algumas variáveis são sobredeterminantes em relação às demais, conforme proposto por Victora et al. (13). Foram levados à análise multivariada todos os fatores cujo $P$ foi igual ou menor a 0,20 (14).
Para o seguimento da coorte, inicialmente os bolsistas foram treinados (40 horas) pelo pesquisador principal na técnica de entrevista e antropometria. O ponto de partida para o seguimento da coorte foi buscar, ativamente, os indivíduos em seus endereços originalmente registrados nas folhas de conglomerado por ocasião da entrevista realizada em 1997. Todos os domicílios registrados foram visitados e a nova entrevista foi realizada quando os indivíduos ainda moravam no endereço original. Se não mais residissem nesses endereços, anotavam-se indicações pertinentes obtidas dos novos moradores, vizinhos ou parentes. Paralelamente foi feito um levantamento nas escolas de ensino fundamental a fim de localizar as crianças não encontradas na busca ativa. Também foram buscados os endereços da mãe ou filhos no registro dos ambulatórios e hospitais da cidade. A localização por meio do serviço de informações de saúde mostrou-se mais efetiva do que a busca nos arquivos escolares porque não há, no município, um banco de dados unificado dos estudantes de ensino fundamental, e foi necessário visitar todas as escolas, particulares, municipais e estaduais. Por outro lado, o registro de atendimento em saúde é bastante completo, pois os dois maiores pontos de atendimento estão concentrados nas duas unidades hospitalares do município (Santa Casa de Misericórdia de Rio Grande e Hospital Universitário).

O controle de qualidade foi feito pela supervisão contínua do trabalho de campo, pela revisão diária dos questionários e pela dupla digitação dos dados. Além disso, 7\% das entrevistas foram repetidas pelo coordenador e pelo supervisor.

\section{RESULTADOS}

Dentre as 775 crianças estudadas em 1997, $412(53,2 \%)$ foram localizadas nos endereços originais. Outras 273 $(35,2 \%)$ foram localizadas em novos endereços, estando 17 em outros municípios. Assim, foram estudados $88,4 \%$ da amostra original (685 crianças), o que corresponde a $11,6 \%$ de perdas.

A distribuição das crianças foi semelhante quanto ao sexo, e $83 \%$ tinham pele de cor branca. A metade das famílias possuía cinco ou mais pessoas, e $6,3 \%$ das mães e $0,2 \%$ dos pais não sabiam ler ou escrever. Uma em cada seis famílias possuía renda mensal igual ou menor a 1 salário mínimo (cerca de US\$ 100). Cerca de 30\% tinham renda mensal menor do que 2 salários mínimos. Setenta por cento das famílias moravam em casas de alvenaria (tijolos) e cerca de $10 \%$ em casas construídas de lata, plástico ou papelão. Praticamente todos os domicílios recebiam água tratada e $95 \%$ possuíam rede de esgoto; $13 \%$ das famílias cozinhavam com fogão a lenha; $75 \%$ das residências não dispunham de qualquer equipamento para o aquecimento doméstico. Em metade dos domicílios havia duas ou mais pessoas por peça, e em $66 \%$ deles três ou mais pessoas dividiam um cômodo para dormir. A maioria das famílias tinha animais domésticos, com a predominância de cães, presentes em $62 \%$ dos domicílios. A prevalência de mães e pais que fumavam, em 1997, foi de 37,7 e 42,6\%, respectivamente. Em 2004, o percentual de mães e pais fumantes foi de 33,5 e 31,5\%, respectivamente.

A prevalência de sibilância recorrente atual foi de 27,9\% (IC95\%: 26,5 a 29,3). Na análise bivariada, estiveram significativamente associadas com sibilância recorrente as seguintes variáveis: aglomeração (pessoa/peça), piso inadequado no domicílio (carpete ou chão batido), aquecimento doméstico inadequado (uso de combustível para aquecimento do ambiente), uso de fogão a lenha para cozinhar, uso de tapetes ou carpete no dormitório, uso de cortinas no dormitório, instalações sanitárias inadequadas (sanitário sem descarga ou fossa negra), realização de menos de seis consultas de pré-natal, baixo peso ao nascer $(<2500 \mathrm{~g})$, antecedente de asma na mãe e nos irmãos, antecedente de rinite nos irmãos, antecedente pessoal de infecção respiratória aguda, história de sibilância anterior e diagnóstico atual de rinite (tabela 1).

A tabela 2 mostra os fatores de risco e proteção identificados na análise 
TABELA 1. Variáveis estudadas e associação com sibilância recorrente, Rio Grande (RS), Brasil, 2004

\begin{tabular}{|c|c|c|c|c|}
\hline Variável & $\begin{array}{c}\text { Sibilância } \\
\text { recorrente } \\
(\%)\end{array}$ & OR & $\mathrm{IC} 95 \%$ & $P$ \\
\hline Escolaridade do pai (anos completos) & & & & 0,11 \\
\hline 9 e mais & 29,5 & 1,00 & & \\
\hline 0 a 8 & 27,1 & 0,89 & 0,61 a 1,28 & \\
\hline Aglomeração (pessoa/peça) & & & & 0,03 \\
\hline Menos de duas & 25,8 & 1,00 & & \\
\hline Duas ou mais & 35,6 & 1,59 & 1,06 a 2,39 & \\
\hline Piso & & & & 0,03 \\
\hline Adequado & 25,1 & 1,00 & & \\
\hline Inadequado (carpete, cimento ou chão batido) & 33,5 & 1,51 & 1,05 a 2,16 & \\
\hline Aquecimento doméstico & & & & 0,002 \\
\hline Adequado & 26,4 & 1,00 & & \\
\hline Inadequado (uso de combustível) & 47,3 & 2,57 & 1,36 a 4,83 & \\
\hline Fogão a lenha para cozinhar & & & & 0,001 \\
\hline Não & 26,0 & 1,00 & & \\
\hline Sim & 45,5 & 2,37 & 1,37 a 4,10 & \\
\hline Tapete ou carpete no dormitório & & & & 0,02 \\
\hline Não & 24,4 & 1,00 & & \\
\hline Sim & 32,6 & 1,50 & 1,06 a 2,13 & \\
\hline Cortinas no dormitório & & & & 0,05 \\
\hline Não & 32,4 & 1,00 & & \\
\hline Sim & 25,1 & 0,69 & 0,49 a 0,98 & \\
\hline Instalação sanitária & & & & 0,04 \\
\hline Adequada & 26,4 & 1,00 & & \\
\hline Inadequada (sanitário sem descarga ou fossa negra) & 37,2 & 1,65 & 1,02 a 2,67 & \\
\hline Número de consultas de pré-natal & & & & 0,008 \\
\hline 6 ou mais & 24,5 & 1,00 & & \\
\hline Menos de 6 & 34,5 & 1,62 & 1,13 a 2,32 & \\
\hline Peso ao nascer & & & & 0,02 \\
\hline $2500 \mathrm{~g}$ ou mais & 26,7 & 1,00 & & \\
\hline Menos de $2500 \mathrm{~g}$ & 43,1 & 2,08 & 1,12 a 3,86 & \\
\hline Aleitamento materno & & & & 0,06 \\
\hline Sim & 26,9 & 1,00 & & \\
\hline Não & 38,2 & 1,68 & 0,97 a 2,91 & \\
\hline Antecedente de asma na mãe & & & & 0,01 \\
\hline Não & 25,7 & 1,00 & & \\
\hline Sim & 37,0 & 1,70 & 1,12 a 2,58 & \\
\hline Antecedente de asma em irmãos & & & & 0,01 \\
\hline Não & 25,6 & 1,00 & & \\
\hline Sim & 37,0 & 1,70 & 1,12 a 2,59 & \\
\hline Antecedente de rinite em irmãos & & & & 0,04 \\
\hline Não & 26,1 & 1,00 & & \\
\hline Sim & 36,0 & 1,59 & 1,02 a 2,49 & \\
\hline Antecedente pessoal de infecção respiratória aguda & & & & 0,004 \\
\hline Não & 22,6 & 1,00 & & \\
\hline $\operatorname{Sim}$ & 32,8 & 1,67 & 1,17 a 2,39 & \\
\hline História de sibilância anterior & & & & 0,04 \\
\hline Não & 24,5 & 1,00 & & \\
\hline Sim & 32,6 & 1,49 & 1,01 a 2,19 & \\
\hline Rinite atual & & & & $<0,0001$ \\
\hline Não & 10,2 & 1,00 & & \\
\hline Sim & 84,1 & 46,87 & 27,47 a 80,50 & \\
\hline Total $(n=685)$ & 27,9 & & & \\
\hline
\end{tabular}

multivariada. O uso de fogão a lenha para cozinhar representou um risco 2,5 vezes maior para ocorrência de sibilância recorrente em relação a quem após controle para diversas variáveis socioeconômicas, ambientais, assistência à gestação e ao parto, aleitamento materno e antecedentes familiares de asma. As crianças que não receberam aleitamento materno tiveram um risco 2 vezes maior de asma $(\mathrm{OR}=2,06$; IC95\%: 1,10 a 3,85). Também houve associação com antecedente de asma em irmãos (OR = 1,95; IC95\% 1,19 a 3,18) e na mãe (OR = 1,80; IC95\% 1,10 a 2,93). Os filhos de mães que realizaram menos de seis consultas de pré-natal tiveram risco $50 \%$ maior de sibilância recorrente, enquanto a escolaridade do pai quando menor de 9 anos completos apareceu como fator de proteção para sibilância recorrente $(\mathrm{OR}=0,65$; IC95\%: 0,42 a 0,98) em relação às crianças cujos pais tinham 9 ou mais anos completos de escolaridade. História de rinite atual mostrou-se associada à sibilância recorrente $(\mathrm{OR}=$ 45,75; IC95\%: 24,19 a 86,52).

\section{DISCUSSÃO}

No presente estudo, alguns fatores relevantes para a sibilância recorrente, como o número de infecções respiratórias e o consumo de antibióticos nos primeiros anos de vida, não foram avaliados, devido à inconsistência dos dados coletados em relação a essas variáveis.

As perdas constituem, possivelmente, a maior limitação dos estudos de coorte em geral. A situação mais freqüente em estudos de base populacional são as perdas por mudança de endereço e, muitas vezes, de cidade (15). Neste estudo, procurarmos minimizar ao máximo o número de indivíduos perdidos através da busca ativa e exaustiva de cada uma das crianças da coorte, inclusive fora do município. Ao analisar comparativamente as 89 crianças perdidas da amostra original, não encontramos diferenças significativas quanto a variáveis demográficas e renda familiar. As crianças diagnosticadas por nós como sibilantes recorrentes e que ainda não estavam sendo tratadas foram encaminhadas ao Serviço de Pneumologia Pediátrica do Hospital Universitário da Fundação Universidade do Rio Grande (FURG). 
TABELA 2. Análise multivariada e relação das variáveis com sibilância recorrente atual, Rio Grande (RS), Brasil, 2004

\begin{tabular}{|c|c|c|}
\hline \multirow[b]{2}{*}{ Variáveis (modelo hierárquico) } & \multicolumn{2}{|c|}{ OR (IC95\%) } \\
\hline & Bruta & Ajustada \\
\hline Fogão a lenha ${ }^{a}$ & $2,37(1,37$ a 4,10$)$ & $2,68(1,44$ a 4,98$)$ \\
\hline TRV ${ }^{b}$ categórico & $P=0,001$ & $P=0,002$ \\
\hline Antecedente pessoal de infecção respiratória aguda ${ }^{c}$ & $1,67(1,17$ a 2,39$)$ & $2,13(1,31$ a 3,48$)$ \\
\hline TRV categórico & $P=0,004$ & $P=0,002$ \\
\hline Aleitamento misto $^{d}$ & $1,68(0,97$ a 2,91$)$ & $2,06(1,10$ a 3,85$)$ \\
\hline TRV categórico & $P=0,06$ & $P=0,02$ \\
\hline Antecedente de asma nos irmãos ${ }^{e}$ & $1,70(1,12$ a 2,59$)$ & $1,95(1,19$ a 3,18$)$ \\
\hline TRV categórico & $P=0,01$ & $P=0,008$ \\
\hline Antecedente de asma na mãe $e^{e}$ & $1,70(1,12$ a 2,58$)$ & $1,80(1,10$ a 2,93$)$ \\
\hline TRV categórico & $P=0,01$ & $P=0,02$ \\
\hline Seis ou mais consultas de pré-natal ${ }^{f}$ & $1,62(1,13$ a 2,32$)$ & $1,57(1,02$ a 2,41$)$ \\
\hline TRV categórico & $P=0,008$ & $P=0,04$ \\
\hline Escolaridade paterna menor de 9 anos completos ${ }^{g}$ & $0,70(0,46$ a 1,08$)$ & $0,65(0,42$ a 0,98$)$ \\
\hline TRV categórico & $P=0,11$ & $P=0,04$ \\
\hline
\end{tabular}

a Ajustada por escolaridade paterna e características ambientais.

b Teste de razão de verossimilhança.

c Ajustada por escolaridade paterna, tipo de fogão, pré-natal, aleitamento materno, antecedentes familiares e pessoais.

d Ajustada por escolaridade paterna, tipo de fogão, pré-natal, variáveis nutricionais e esquema vacinal.

e Ajustada por escolaridade paterna, tipo de fogão, pré-natal, aleitamento materno e antecedentes familiares.

${ }^{f}$ Ajustada por escolaridade paterna, tipo de fogão e características pré-natais e do parto.

9 Ajustada por sexo, escolaridade materna e renda familiar.

A coleta de dados do seguimento da coorte foi feita durante todo o ano de 2004. É possível considerar que fatores climáticos possam ter influenciado parte dos resultados, uma vez que o aspecto sazonal é importante no comportamento epidemiológico das doenças sibilantes (16). No entanto, como o período utilizado no recordatório foi de 12 meses, a influência de possíveis fatores climáticos deve ter sido muito pequena.

A limitação mais importante deste estudo é, provavelmente, o critério diagnóstico de sibilância recorrente. Para a obtenção deste diagnóstico foram adaptadas questões já amplamente utilizadas e validadas em outras pesquisas sobre doenças respiratórias, tendo por base o questionário ATS-DLD-78 C (11). A caracterização de sibilância recorrente atual foi bastante objetiva; no entanto, observa-se que o diagnóstico epidemiológico apresenta alta sensibilidade mas baixa especificidade, não apenas pela forma da coleta dos dados, mas também pela ampla possibilidade de encontrar sibilos relacionados a diversas patologias respiratórias (17). É preciso considerar, ainda, que, ao medir a sibilância reco- rrente atual, certamente lidamos com dois tipos de sibilantes: crianças que começaram a sibilar nos primeiros anos de vida e continuam com o sintoma (sibilantes persistentes) e crianças que começaram a sibilar mais tarde, a partir dos 5 anos (sibilantes tardios). Em comparação à coorte de Martinez (18), o grupo de sibilantes persistentes será provavelmente o grupo com atopia, conforme a classificação de Stein et al. (19) e como demonstram diversos estudos. Quanto aos sibilantes tardios, considerando a faixa etária dos mesmos, provavelmente poderiam ser rotulados como portadores de asma, seguindo os critérios do estudo internacional ISAAC, que considera o período de 12 meses anteriores para o diagnóstico (20).

Neste estudo houve associação estatisticamente significativa de sibilância recorrente com uso de fogão a lenha, um achado consistente com os resultados de outros estudos $(21,22)$. Por outro lado, poucos estudos sobre asma e sibilância têm mostrado associação significativa com o número de consultas de pré-natal realizadas pelas mães. Há evidências de que a capacidade de resposta imunológica inicia na vida intra-uterina, sendo que a alergia materna e outras exposições podem afetar esse processo antes do nascimento. As mães que não realizam regularmente acompanhamento pré-natal podem estar mais suscetíveis a essas situações (23). Neste estudo, os filhos de mães que não fizeram o esquema de consultas de pré-natal recomendado pela Organização Mundial da Saúde (OMS) apresentaram mais sibilância recorrente atual, como já apontado em estudos anteriores $(23,24)$.

Observou-se ainda associação inversa entre o aleitamento materno e a ocorrência de sibilância recorrente atual. O efeito ficou evidente após a inclusão da variável no modelo de regressão logística: houve 2 vezes mais risco de sibilância recorrente atual nas crianças que não foram amamentadas ao seio. Esse achado não é consistente com a teoria da higiene, que sugere que as crianças com aleitamento exclusivo estão mais sujeitas ao desenvolvimento de atopia (25). Contudo, é possível que as doenças sibilantes e a atopia estejam associadas de forma independente com a asma na infância, e que seus efeitos sejam mediados por diferentes mecanismos, sendo o aleitamento materno um fator de proteção tanto para infecções respiratórias quanto para desenvolvimento de asma na criança (26).

Algumas variáveis relacionadas com antecedentes familiares neste estudo mostraram associação com sibilância recorrente atual. A história de asma na mãe e nos irmãos, assim como a rinite nos irmãos, estiveram associadas com sibilância recorrente atual na análise bivariada. Assim como em diversos estudos, a asma na mãe e nos irmãos manteve a associação com sibilância recorrente atual após ajuste para fatores socioeconômicos, ambientais, pré-natais e nutricionais $(27,28)$. Há quase 2 vezes mais risco de sibilância recorrente atual para crianças cuja mãe ou irmãos apresentam história de asma.

Alguns estudos mostram que a sibilância nos primeiros anos de vida, principalmente provocada pelo vírus sincicial respiratório, é um fator preditivo de asma futura (29), embora muitos 
estudos sugiram uma relação inversa entre infecções respiratórias prévias e o risco de asma (30). No presente estudo, encontrou-se associação significativa entre episódios anteriores de infecções respiratórias agudas e sibilância recorrente atual. Não foi possível estabelecer a etiologia das infecções relatadas. Além disso, é possível supor que muitas ocorrências registradas como infecções respiratórias agudas tenham sido manifestações precoces de hiper-reatividade brônquica.

Neste estudo foi encontrada fortíssima associação entre rinite e sibilância recorrente atual, sendo o risco de sibilância recorrente 45 vezes maior nas crianças com diagnóstico de rinite. Assim como este, muitos estudos têm fornecido evidências de uma forte associação entre rinite e asma, sugerindo a hipótese de que a rinite alérgica e a asma são manifestações da mesma doença $(31,32)$. Trabalhos recentes têm mostrado que os fatores de risco são comuns tanto à rinite quanto à asma. É possível que a asma sem rinite seja uma condição diferente, pelo menos com respeito a alguns fatores de risco relevantes (33).

Como a amostra estudada é representativa, os resultados podem ser extrapolados para a população dessa faixa etária da Cidade de Rio Grande. É provável ainda que, em função da semelhança entre os municípios do extremo sul do Brasil em termos socioeconômicos, estes mesmos achados possam ser extrapolados para a grande maioria deles.

Os resultados aqui apresentados têm implicações de interesse clínico e epidemiológico. A forte associação entre rinite e sibilância recorrente indica que o manejo individual da asma deve contemplar a investigação e, pos- sivelmente, o tratamento conjunto de rinite. Por sua ocorrência bastante elevada, a sibilância recorrente é um importante problema de saúde pública e, como tal, são necessárias ações apropriadas para o seu controle e tratamento. É possível estabelecer estratégias de planejamento em saúde pública no sentido de minimizar os efeitos dessa situação, especialmente com o desenvolvimento de programas educativos e terapêuticos para a asma. Tais programas reduziriam, em muito, os gastos com sibilância recorrente, sobretudo hospitalares, e contribuiriam de forma efetiva para a melhoria da qualidade de vida de uma parcela considerável da população.

Agradecimentos. Este estudo foi financiado pela Prefeitura Municipal de Rio Grande e pela Fundação Universidade Federal do Rio Grande.

\section{REFERÊNCIAS}

1. Phelan PD, Olinsky A, Robertson CF. Respiratory illness in children. $4^{\mathrm{a}}$ ed. Oxford: Blackwell Scientific Publication; 1994.

2. Robertson CF, Sennhauser FH, Mallol J. Alteração na incidência e na gravidade da asma, nos países desenvolvidos e em desenvolvimento. Em: Phenan PD, editor. Baillière's Clinical Paediatrics. Vol. 3. London: Baillière Tindall, 1995; Pp. 253-73.

3. Worldwide variations in the prevalence of asthma symptoms: the International Study of Asthma and Allergies in Childhood (ISAAC). Eur Respir J. 1998;12(2):315-35.

4. Mallol J, Sole D, Asher I, Clayton T, Stein R, Soto-Quiroz M. Prevalence of asthma symptoms in Latin America: the International Study of Asthma and Allergies in Childhood (ISAAC). Pediatr Pulmonol. 2000;30(6): 439-44.

5. Werneck G, Ruiz S, Hart R, White M, Romieu I. Prevalence of asthma and other childhood allergies in Brazilian schoolchildren. J Asthma. 1999;36(8):677-90.

6. Sole D, Yamada E, Vana AT, Werneck G, Solano de Freitas L, Sologuren MJ, et al. International study of asthma and allergies in childhood (ISAAC): prevalence of asthma and asthma-related symptoms among Brazilian schoolchildren. J Investig Allergol Clin Immunol. 2001;11(2):123-8.

7. Teldeschi AL, Sant'anna CC, Aires VL. Prevalence of respiratory symptoms and clinical conditions and associated asthma in schoolchildren in Rio de Janeiro, Brazil. Rev Assoc Med Bras. 2002;48(1):54-9.
8. Chatkin MN, Menezes AM. Prevalence and risk factors for asthma in schoolchildren in southern Brazil. J Pediatr (Rio J). 2005;81(5): 411-6.

9. Prietsch SO, Fischer GB, Cesar JA, Lempek BS, Barbosa LV Jr, Zogbi L, et al. Doença respiratória em menores de 5 anos no sul do Brasil: influência do ambiente doméstico. Rev Panam Salud Publica. 2003;13(5):303-10.

10. von Mutius E. The burden of childhood asthma. Arch Dis Chid. 2000;82(Suppl 2): II2-5.

11. Ferris BG. Epidemiology Standardization Project (American Thoracic Society. Am Rev Respir Dis. 1978;118(6 Pt 2):1-120.

12. Rothman KJ. Modern epidemiology. Boston: Little, Brown and Co.; 1986.

13. Victora CG, Huttly SR, Fuchs SC, Olinto MTA. The role of conceptual frameworks in epidemiological analysis: A hierarchical approach. Intern J Epidemiol. 1997;26(1):224-7.

14. Mickey RM, Greenland S. The impact of confounder selection criteria on effect estimation. Am J Epidemiol. 1989;129(1):125-37.

15. Grimes DA, Schulz KF. Cohort studies: marching towards outcomes. Lancet. 2002;359 (9303):341-5.

16. Hashimoto M, Fukuda T, Shimizu T, Watanabe S, Watanuki S, Eto Y, et al. Influence of climate factors on emergency visits for childhood asthma attack. Pediatr Int. 2004;46(1): $48-52$.

17. Joseph CL, Foxman B, Leickly Fe, Peterson E, Ownby D. Sensitivity and specificity of asthma definitions and symptoms used in a survey of childhood asthma. J Asthma. 1999; 36(7):565-73.

18. Martinez FD. Development of wheezing disorders and asthma in preschool children. Pediatrics. 2002;109(2 Suppl):362-7.

19. Stein RT, Holberg CJ, Morgan WJ, Wright AL, Lombardi E, Taussig L, et al. Peak flow variability, methacholine responsiveness and atopy as markers for detecting different wheezing phenotypes in childhood. Thorax. 1997;52(11):946-52.

20. Ellwood P, Asher MI, Beasley R, Clayton TO, Stewart AW, ISAAC Steering Committee. The international study of asthma and allergies in childhood (ISAAC): phase three rationale and methods. Int J Tuberc Lung Dis. 2005;9(1): 10-6.

21. Schei MA, Hessen JO, Smith KR, Bruce N, McCracken J, Lopez V. Childhood asthma and indoor woodsmoke from cooking in Guatemala. J Expo Anal Environ Epidemiol. 2004; 14(Suppl 1):S110-7.

22. Kilpelainen $M$, Koskenvuo $M$, Helenius $H_{r}$ Terho E. Wood stove heating, asthma and allergies. Respir Med. 2001;95(11):911-6.

23. Oliveti JF, Kercsmar CM, Redline S. Pre- and perinatal risk factors for asthma in inner city African-American children. Am J Epidemiol. 1996;143(6):570-7.

24. Hanrahan JP, Halonen M. Antenatal interventions in childhood asthma. Eur Respir J. 1998;12(Suppl 27):46s-51s.

25. Strachan DP. Family size, infection and atopy: the first decade of the "hygiene hypothesis". Thorax. 2000;55(Suppl 1):S2-10. 
26. Oddy WH. A review of the effects of breastfeeding on respiratory infections, atopy, and childhood asthma. J Asthma. 2004;41(6): 605-21.

27. Ehrlich RI, Du Toit D, Jordaan E, Zwarenstein M, Potter P, Volmink JA, et al. Risk factors for childhood asthma and wheezing. Importance of maternal and household smoking. Am J Respir Crit Care Med. 1996;154(3 Pt 1):681-8.

28. Alford SH, Zoratti E, Peterson EL, Maliarik M, Ownby DR, Johnson CC. Parental history of atopic disease: disease pattern and risk of pediatric atopy in offspring. J Allergy Clin Immunol. 2004;114(5):1046-50.
29. Stein RT, Sherrill D, Morgan WJ, Holberg CJ, Halonen M, Taussig LM, et al. Respiratory syncytial virus in early life and risk of wheeze and allergy by age 13 years. Lancet. 1999; 354(9178):541-5.

30. Remes ST, Korppi M. On roots of childhood asthma: the role of respiratory infections. Ann Med. 2005;37(1):26-32.

31. Bugiani M, Carosso A, Migliore E, Piccioni P, Corsico A, Olivieri M, et al. Allergic rhinitis and asthma comorbidity in a survey of young adults in Italy. Allergy. 2005;60(2):165-70.
32. Smart BA, Slavin RG. Rhinosinusitis and pediatric asthma. Immunol Allergy Clin North Am. 2005;25(1):67-82.

33. Sherrill DL, Guerra S, Cristina Minervini M, Wright AL, Martinez FD. The relation of rhinitis to recurrent cough and wheezing: a longitudinal study. Respir Med. 2005;99(11): 1377-85.

Manuscrito recebido em 2 de março de 2006. Aceito em versão revisada em 6 de julho de 2006.

ABSTRACT Objective. To study the prevalence of and major factors associated with recurrent wheezing in children younger than 13 years of age in the urban area of Rio Grande, in the state of Rio Grande do Sul, Brazil.

Risk factors for recurrent wheezing in children under 13 years old in the South of Brazil

Methods. The presence of recurrent wheezing was investigated in a cohort as part of a cross-sectional study that was begun in 1997 that focused on the morbidity from respiratory diseases in children then between 0 and 5 years of age. During home visits in 2004 a standardized questionnaire given by trained interviewers was used to obtain information concerning the family's socioeconomic and living conditions, maternal care during pregnancy and delivery, and children's current and previous morbidity patterns. The statistical analysis included the calculation of the odds ratio (OR) and $95 \%$ confidence interval $(95 \% \mathrm{CI})$, with nonconditional logistic regression adjustment for potential confounding factors, according to a predefined hierarchical model.

Results. Of the 775 children studied in 1997, 685 were located in 2004 (loss of 11.6\%). In this group, the prevalence of recurrent wheezing at the time of the interview was 27.9\%. After adjustment, the risk factors were: current rhinitis $(\mathrm{OR}=45.7 ; 95 \% \mathrm{CI}: 24.2$ to 86.5$)$, use of wood stove for cooking $(\mathrm{OR}=2.7 ; 95 \% \mathrm{CI}$ : 1.4 to 4.9$)$, child's history of acute respiratory infection $(\mathrm{OR}=2.1 ; 95 \% \mathrm{CI}$ : 1.3 to 3.5$)$, bottle feeding $(\mathrm{OR}=2.1 ; 95 \%$ CI: 1.1 to 3.8), history of asthma in siblings (OR $=1.9 ; 95 \% \mathrm{CI}: 1.2$ to 3.2$)$, maternal history of asthma (OR $=1.8 ; 95 \% \mathrm{CI}$ : 1.1 to 2.9$)$, and fewer than six prenatal medical consultations (OR $=1.6 ; 95 \% \mathrm{CI}$ : 1.1 to 2.4$)$. Paternal schooling $<9$ years was a protective factor against recurrent wheezing $(\mathrm{OR}=0.6 ; 95 \% \mathrm{CI}$ : 0.4 to 0.9$)$.

Conclusions. These results suggest that the management of recurrent wheezing and asthma must consider checking for and simultaneously treating rhinitis. The measures to minimize the effects of recurrent wheezing should include educational and treatment programs focusing on asthma.

Key words Asthma, child, epidemiology, risk factors, respiratory sounds, Brazil. 